\title{
Advanced methods for uncertainty assessment and global sensitivity analysis of a Eulerian atmospheric chemistry transport model
}

\author{
Ksenia Aleksankina, ${ }^{1,2}$ Stefan Reis ${ }^{2,3}$, Massimo Vieno ${ }^{2}$, and Mathew R. Heal ${ }^{1}$ \\ ${ }^{1}$ School of Chemistry, University of Edinburgh, Edinburgh, UK \\ ${ }^{2}$ NERC Centre for Ecology \& Hydrology, Penicuik, UK \\ ${ }^{3}$ University of Exeter Medical School, European Centre for Environment and Health, Knowledge Spa, Truro, UK
}

Figure S1 shows the spatial distribution of $k$-fold cross-validation errors for the Gaussian process emulators used to estimate the annual average surface concentrations of $\mathrm{O}_{3}, \mathrm{NO}_{2}$, and $\mathrm{PM}_{2.5}$. The $k$-fold cross-validation values were calculated as described by Lataniotis et al. (2017)
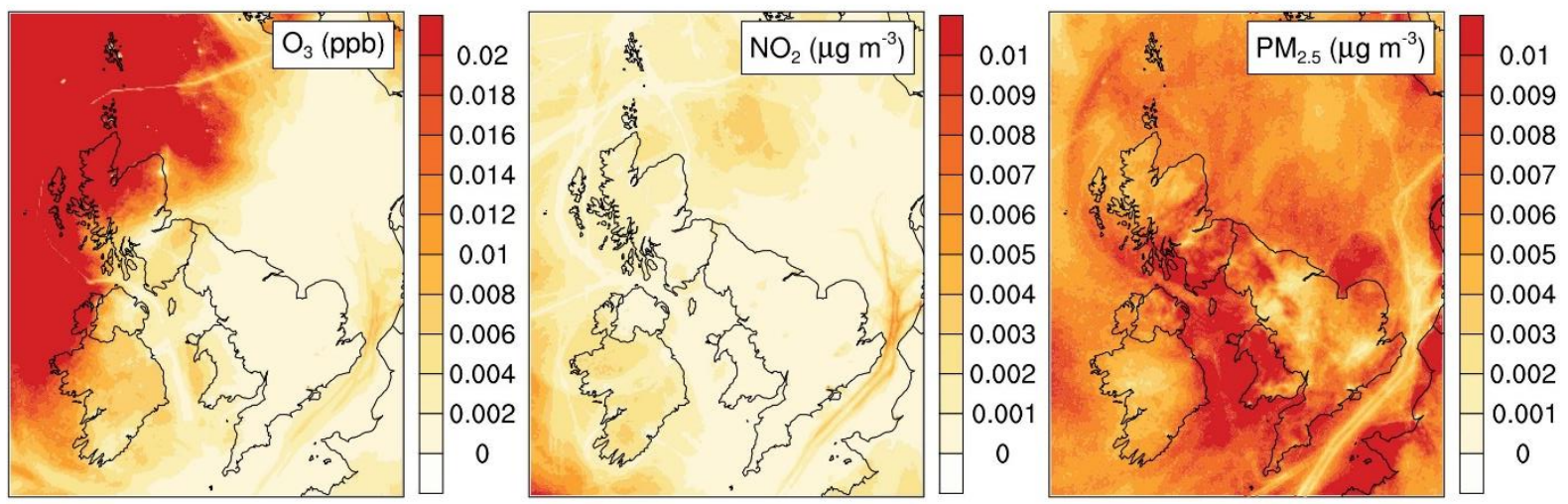

Figure S1 Spatial distribution of $\boldsymbol{k}$ - fold cross validation error values for emulated annual average concentrations of $\mathrm{O}_{3}, \mathrm{NO}_{2}$, and $\mathrm{PM}_{2.5}$.

Figure S2 shows the scatter plot of the first-order sensitivity indices against the total sensitivity indices. The grey lines indicate $\pm 3 \%$ fluctuation in the sensitivity index values, which were attributed to numerical errors in the calculation of sensitivity indices the analytical values of which are close to zero. 


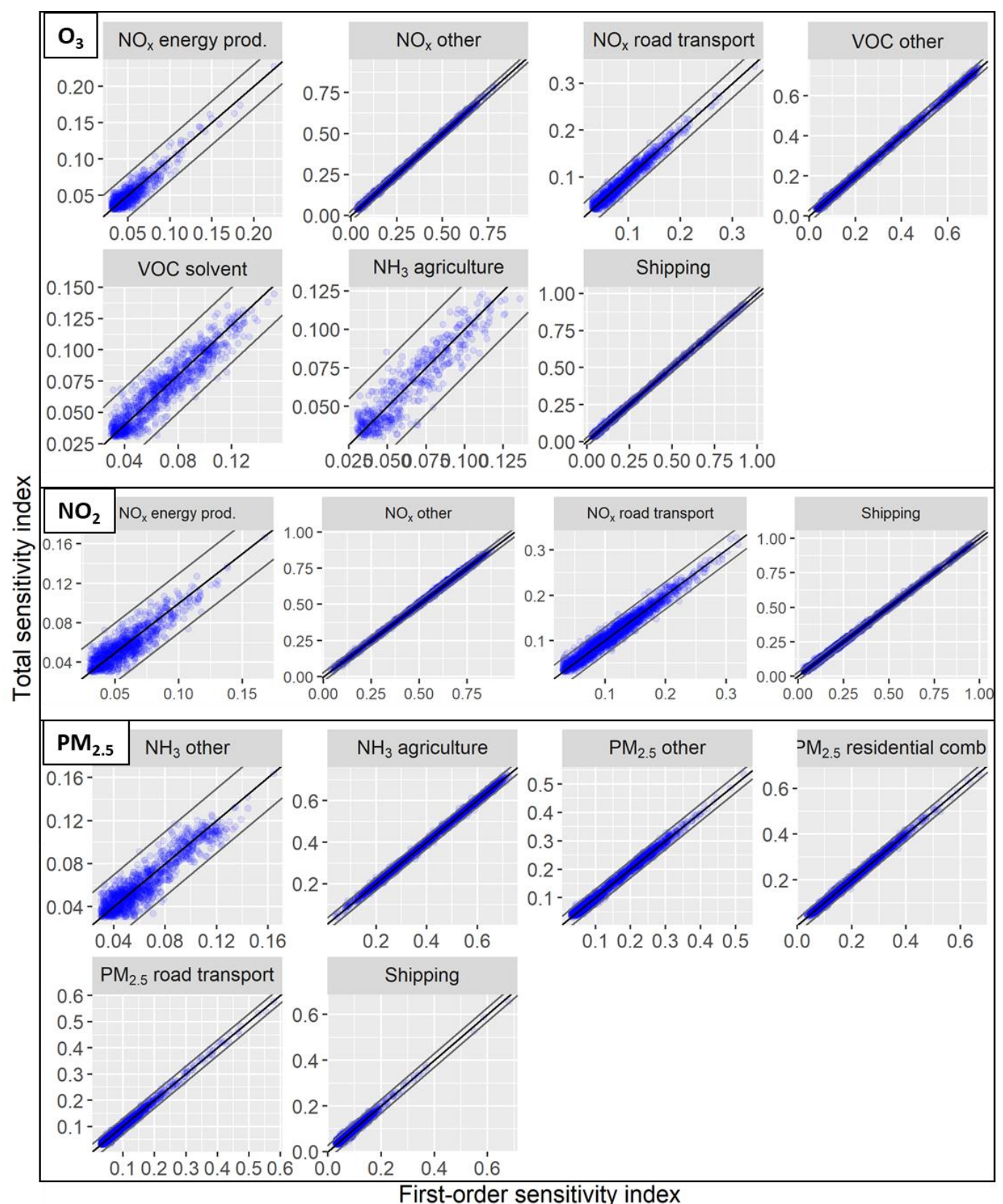

Figure S2 Scaterplot of the first-order sensitivity indices against the total sensitivity indices for the inputs affecting the variation in modelled values of $\mathrm{O}_{3}, \mathrm{NO}_{2}$, and $\mathrm{PM}_{2.5}$. 